\title{
Origin and distribution of volatile elements in the early Solar System
}

\begin{abstract}
E. FÜRI ${ }^{1}$
${ }^{1}$ CRPG, CNRS - Université de Lorraine, Vandœuvre-lesNancy, France (efueri@crpg.cnrs-nancy.fr)

Tracing the origin and distribution of volatile elements in the early Solar System is key for understanding the source(s) of water and the building blocks of life on Earth and other planetary bodies. During the protoplanetary disk phase, volatile species $\left(\mathrm{H}_{2}, \mathrm{H}_{2} \mathrm{O}, \mathrm{He}, \mathrm{CO}, \mathrm{CO}_{2}, \mathrm{CH}_{4}, \mathrm{~N}_{2}, \mathrm{NH}_{3}, \mathrm{H}_{2} \mathrm{~S}\right.$, etc.) resided in the gas phase throughout a significant portion of the disk; however, at sufficiently low temperatures, volatile ices played a key role in planet formation by promoting coagulation of ice-coated dust grains into larger pebbles. Thus, based on predictions of a strong heliocentric radial gradient in temperature, the volatile content and composition of different planetesimals should have been characterized by sharp transitions between species that were refractory enough to freeze out, and those that were too volatile and remained in the gas phase. Planetesimals that accreted close to the Sun are expected to by dry and C-poor, whereas bodies that formed in the outer Solar System should have accreted H-C-N-rich ices and organics. Even as the temperature decreased below the water condensation threshold, proto-Jupiter may have prevented the inward drift of ice particles [1]. This is consistent with the notion that Jupiter acted as a barrier against the transport of material from the outer towards the inner Solar System, and thus caused the spatial separation between the "non-carbonaceous" (NC) and "carbonaceous" (CC) Cr-Ti-O-Mo-Ru isotope reservoirs [e.g., 2-4].

However, Earth's H, N, O, and Mo-Ru isotope signatures require the terrestrial building blocks to have included both NC- and CC-type bodies [4,5]. A contribution of CC-type (CI- and/or CM-like) material may also be required to explain the H-N isotopic composition of Mars and the Moon $[5,6]$. This implies that the NC and CC reservoirs communicated with each other during the main stages of terrestrial planet formation. Importantly, the $\mathrm{H}-\mathrm{N}$ isotope signature of mineralhosted melt inclusions in the angrite D'Orbigny imply that nitrogen- and water-rich objects that formed in the outer Solar System, beyond the orbit of Jupiter, were delivered to the terrestrial planet-forming region with the first $\sim 4$ Myr after the formation of Ca-Al-rich inclusions [8].
\end{abstract}

[1] Morbidelli et al. (2016) Icarus 267, 368-376. [2] Kruijer et al. (2017) PNAS 114, 6712-6716. [3] Warren et al. (2011) EPSL 311, 93-100. [4] Hopp et al. (2020) EPSL 534, 116065.

[5] Alexander (2017) Philos. T. Roy. Soc. A 375, 20150284. [6] Füri et al. (2015) EPSL 431, 195-205. [7] Füri et al. (2017) EPSL 474, 76-82. [8] Deligny et al., in revision. 